\title{
Using an Adaptation of Piaget's Operative Logic of Classes for Analyzing Classification Systems
}

\author{
Raul Sidnei Wazlawick \\ Universidade Federal de Santa Catarina \\ Program de Doutorado em Engenharia de Producao \\ GRUPPO - DCEC \\ Florianopolis, Brazil
}

\begin{abstract}
The main goal of this paper is to show a useful application of genetic psychology to artificial intelligence. It shows a way to interpret some concepts of classification structures by utilizing operative logic of classes defined by Jean Piaget. This paper will show that classification systems can belong to one of three distinct stages: taxonomies, simple classifications and classifications with exceptions. Each of these stages will be analyzed through the same framework utilized by Piaget for analyzing human knowledge.
\end{abstract}

\section{INTRODUCTION}

Genetic psychology is a science that studies the development of human knowledge. The main goal of this paper is to show a useful application of genetic psychology to artificial intelligence. Specifically, it shows a way to interpret some concepts of classification structures utilizing an adaptation of Piaget's operative logic of classes.

In speaking of intelligent computational systems we can have a notion of development, because if a computer can "know" something, that knowledge is developed through an interaction with an environment. Therefore, utilizing some concepts of genetic psychology may be useful for analyzing development in intelligent computational systems. This paper is restricted to a certain area of artificial intelligence, called "classification systems" or "inheritance theory." It will be briefly developed in section 2 .

Classification systems can belong to one of three distinct stages: taxonomies, simple classifications and classifications with exceptions. Each of these stages will be analyzed through the same framework utilized by Piaget for analyzing human knowledge.

In section 3, the framework for analyzing classification systems will be very briefly introduced and summarized. That framework is a version of Piaget's operative logic of classes, extended by this author with two epistemic operators.

In section 4, the analysis itself will be carried out. The main properties and differences among simple inheritance, taxonomy and inheritance with exceptions will be demonstrated.

\section{CLASSIFICATION SYSTEMS}

It is possible to define a class as a collection of individuals with certain shared attributes. For example, the class of vertebrates is the collection of individuals that have a vertebral column. Thus, that class is characterized by the attribute "has a vertebral column." 
Further, a class " $X$ " may be defined as a collection where every individual of " $X$ " belongs to the class " $Y$ ", but where every individual of " $X$ " has an attribute that is not shared by every individual of " $Y$ ". Such a definition is by genus and specific difference. In such a case " $X$ " is a subclass of " $Y$ ", and " $X$ " inherits attributes from " $Y$ ". The members of " $Y$ " that don't belong to " $X$ " will belong to its complementary, called " $X$ ", where $X^{\prime}=Y-X$.

For example, let " $\mathrm{X}$ " be the class of mammals, and " $\mathrm{Y}$ " be the class of vertebrates. Then " $\mathrm{X}$ " is the class of vertebrates that are not mammals (including fishes, birds, etc.). Following this example, " $X$ " inherits attributes from " $Y$ " because every mammal also has a vertebral column. This kind of inheritance is called "positive simple inheritance."

Graphically:

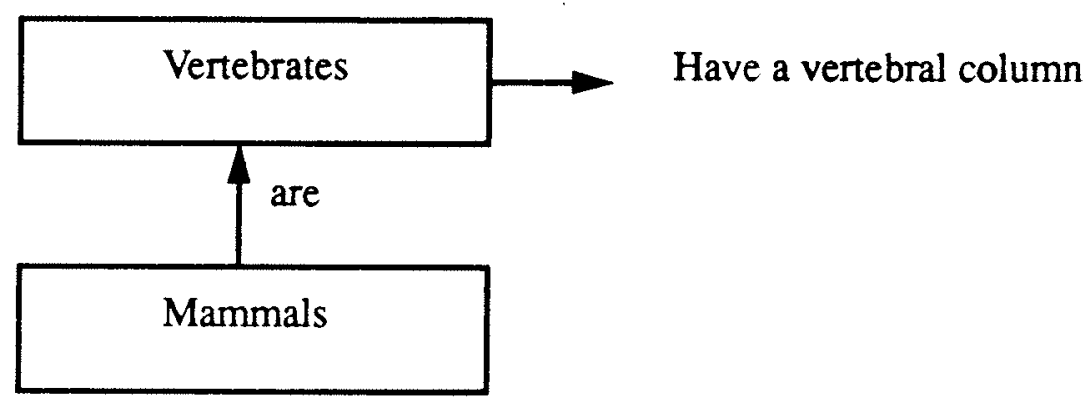

If classes " $X$ " and " $Y$ " don't share any member, there exists a negative simple inheritance among them. In such a case, " $X$ " inherits the negation of the attributes of " $Y$ " (and vice versa).

For example:

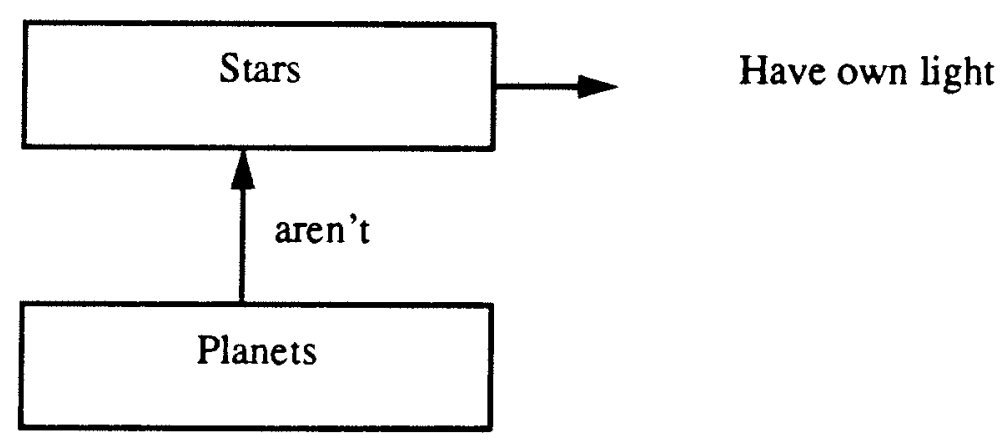

Therefore, planets do inherit the attribute "don't have own light".

As a shorthand, positive simple inheritance will be represented by $(X \rightarrow Y)$, for example, (Mammal $\rightarrow$ Vertebrate), and negative simple inheritance relations will be represented by $(X \rightarrow Y)$, for example, (Planet $\rightarrow$ Star).

Positive simple inheritance between classes " $\mathrm{X}$ " and " $\mathrm{Y}$ ", is the same as inclusion of sets $X$ and $Y$, composed of the members of " $X$ " and " $Y$ " and is expressed as: $(X \subseteq Y)$; and negative simple 
inheritance between " $X$ " and " $Y$ " is the same as saying that $X$ belongs to the complementary set of $Y$, expressed as: $(X \subseteq Y)$.

From the examples it can be seen that simple inheritance doesn't admit exceptions to classification rules. If mammals are vertebrates, every mammal is a vertebrate. Another kind of inheritance relation is one that admits exceptions to classification rules. That kind of relation also admits positive and negative cases. A case of inheritance with exceptions that can't be modelled by simple inheritance is the generic statement "birds are fliers." Though it is true for a typical bird, there are some exceptions, such as penguins.

Evidently, that kind of relation doesn't have a simple interpretation in terms of set inclusion. It would be inconsistent in terms of set inclusion. It would be inconsistent to assert: (Penguin $\rightarrow$ Bird), (Bird $\rightarrow$ Flier) and (Penguin $\rightarrow$ Flier), because there isn't any set that satisfies those assertions together. The only way this could be the case would be if the class of penguins were equivalent to an emptyset. This is not, evidently, desirable. Inheritance with exceptions must, therefore, be analyzed with a non-monotonic logic, where new assertions can preempt old conclusions.

\section{OPERATIVE LOGIC OF CLASSES}

Now, operative logic of classes will be introduced. It has eight different forms. Its simplest form, called "Grouping I,"permits operations on the following structure:

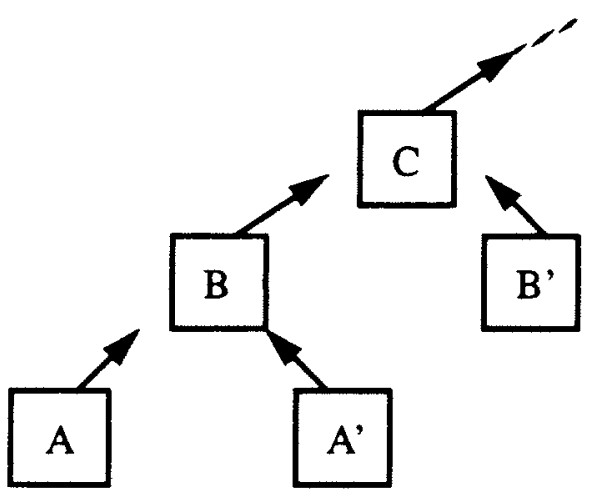

Here, letters denote classes, and arrows denote positive simple inheritance. Furthermore, $A$ and $A^{\prime}$ are a partition of $\mathrm{B} ; \mathrm{B}$ and $\mathrm{B}^{\prime}$ are a partition of $\mathrm{C}$, and so forth.

It is possible to define addition and subtraction operations on contiguous classes. Two classes " $\mathrm{X}$ " and " $Y$ " are contiguous if they are included in the same class " $\mathrm{Z}$ ", or if one of them is included in the other one, or if they are the same class, or yet if one of them is empty $(\varnothing)$. The following kinds of operations are identified:

1) Direct: $A+A^{\prime}=B, B+B^{\prime}=C$, etc.

2) Inverse: $B-A=A^{\prime}, B-A^{\prime}=A$, etc.

3) Tautological: $A+A=A, B+B=B$, etc.

4) Reabsorbent: $A+B=B, A^{\prime}+B=B$, etc.

5) Cancellation: $A-A=\varnothing, B-B=\varnothing$, etc.

6) Neutral: $A+\varnothing=A, B-\varnothing=B$, etc. 
A second form of logic of classes, called Grouping II, admits the composition of several structures of the previous kind. For example, let $A_{1}+A_{1}=B_{1}$ and $A_{2}+A_{2}=B_{1}$. Then, it's possible to compound these expressions, and build a structure called "vicariancy" that has the form: $B_{1}=A_{1}+A_{1^{\prime}}=A_{2}+A_{2^{\prime}}$.

In such a case, if $B_{1}$ is the class of mammals, $A_{1}$ may be the class of dogs, and $A_{2}$ the class of cats. Thus, $A_{1}$ ' will be the class of mammals that are not dogs, and $A_{2}$ the class of mammals that are not cats. For this grouping, classes $A_{1}$ and $A_{2}$ must be disjoint. Such a restriction doesn't apply, however, to classes $A_{1}$, and $A_{2}$. Note that the classes of not-dogs and not-cats are not disjoint; their intersection can contain mice, elephants, etc.

The last form that will be introduced here is Grouping IV, which consists of comparing or multiplying two or more classifications. Grouping III will be omitted because it's not central to this presentation. Groupings V to VIII will be omitted for the same reason, since they belong to the operative logic of relations. Thus, let $B_{1}$ and $B_{2}$ be two classes with exactly the same members, but where $B_{1}=A_{1}+A_{1^{\prime}}$ and $B_{2}=A_{2}+A_{2^{\prime}}$. Then $B_{1} \times B_{2}=A_{1} \times A_{2}+A_{1} \times A_{2}^{\prime}+A_{1^{\prime}} \times A_{2}+A_{1^{\prime}} \times A_{2}$. The symbol " $x$ " indicates an intersection (or multiplication) operation on classes.

The following graphic should help clarify Grouping IV:

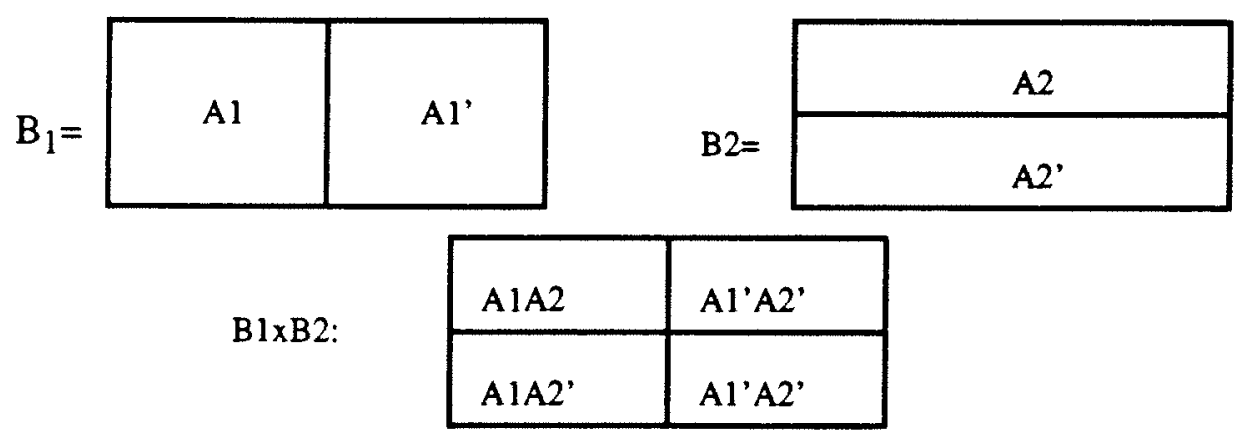

For example, let $B_{1}$ be the class of humans, divided into adults and not-adults, and $B_{2}$ be the class of humans divided into women and not-women. Then $\mathrm{B}_{1} \mathrm{xB}_{2}$, or in other words $\mathrm{B}_{1}$ compared to $B_{2}$, is equivalent to a set of the following subclasses: $\left.i\right)$ adult women, ii) non-adult women, iii) adult not-women, and iv) non-adult not-women.

Finally, to these Groupings we can add an epistemic operator on classes $k$, and an epistemic operator on equations $\mathcal{X}$. An epistemic operator indicates the knowledge that one can have about an object (in this case: a class or an equation). Here $x(A)$ gives a known subclass of $A$, and $\mathcal{X}\left(A_{1} \times A_{2}=A_{1} A_{2}\right)$ means that it is known that the intersection among $A_{1}$ and $A_{2}$ is not empty.

Some examples of writing expressions utilizing operative logic extended with these epistemic operators will be given. The metalanguage utilized is first order predicate calculus with some wellknown predicates of set theory. 
As $k(A)$ is a known subclass of $A$, then one property of $k$ is: "for all $X, k(X) \subseteq X$ ". Then, it can be derived, by properties of Grouping I that for the expression "for all $X$ and $Y, k(X)+(\kappa(X))^{\prime}=k(Y)$ implies $X+X^{\prime}=Y^{\prime \prime}$. One property of $\mathcal{X}$ is: the expression "for all $X$ and $\left.Y, X X X Y=X Y\right)$ implies $\mathrm{XxY}=\mathrm{XY} \mathrm{Y}^{\prime \prime}$.

$K$ and $\mathcal{X}$ are not features of original operative logic. They are an extension, developed by the author, for applications on commonsense knowledge. The original version of Piaget was thought to be for the analysis of scientific knowledge only. For example, according to Piaget's logic, Dogs+Dogs' =Mammals' =Animals can't be compounded with Dogs+Dogs'=Mammals without contradiction, because, in Grouping II Dogs+Dogs'=Mammals+Mammals'=Animals implies that Dogs $\subseteq$ Mammals', and, thus, Dogs can't be Mammals. But by utilizing epistemic operators it's possible to get free from this problem. This will be shown in the example in section 4.2.

\section{ANALYZING CLASSIFICATION SYSTEMS USING OPERATIVE LOGIC OF CLASSES EXTENDED BY $\kappa$ AND $\mathcal{K}$}

The development of three forms of classification systems will be analyzed: taxonomies, simple classification and classification with exceptions. Taxonomies correspond to scientific knowledge fully systematized. Simple classifications are also related to scientific knowledge, but the knowledge is not fully systematized; it is incomplete knowledge, that grows during interaction with the environment. Classification with exceptions is very far from scientific knowledge, but it is closer to natural or commonsense knowledge.

\subsection{Taxonomies}

A taxonomy is a structure employed in biology for describing animal and plant classification. It's assumed that if a class is divided into $n$ subclasses, every subclass appears in the taxonomic schema (a taxonomy is complete). Furthermore, classes at the same level of hierarchy are necessarily disjoint, and if classes " $X$ " and " $Y$ " are not in a relationship $X \subseteq Y$ or $Y \subseteq X$, then " $X$ " and " $Y$ " are disjoint.

Inheritance relations on taxonomies correspond exactly to the structure of Grouping II. For doing the calculus of Grouping II, it is necessary that any classes at the same level be disjoint and every class be exhaustive. For instance, let $B_{1}$ be divided in $A_{1}, A_{2}, \ldots, A_{n}$. Then $A_{1} \times A_{2}=\varnothing, A_{1} \times A_{3}=\varnothing, \ldots$, $A_{n-1} \times A_{n}=\varnothing$, and $A_{1}+A_{2}+\ldots+A_{n}=B_{1}$.

On a taxonomy, one can say that "for all $X$ and $Y, X+X^{\prime}=Y+Y^{\prime}$ implies $X \subseteq Y^{\prime}$ and $Y \subseteq X^{\prime}$." That is valid only if knowledge is complete, i.e. , if $\kappa(X x Y=\varnothing)$, which may be read as "it's known that $\mathrm{X}$ and $\mathrm{Y}$ are disjoint, i.e. that their intersection is equivalent to the empty class.

\subsection{Simple Classification}

A simple classification is like a taxonomy but does not meet disjunction and exhaustivity conditions for classes occurring at the same level of hierarchy. This happens because knowledge is not complete, and one can't say that "for all $X$ and $Y$, if $X+X^{\prime}=Y+Y^{\prime}=Z$ implies $X x Y=\varnothing$." Simple 
classification is the most common case for situations in which classification systems (without exceptions) grow and develop with knowledge acquisition.

Consider a classification system with the following rules: (Dogs $\rightarrow$ Animals) and (Mammals $\rightarrow$ Animals). The system doesn't know whether dogs are mammals, because this information was not yet provided (but, it will be, possibly, in the future). At this stage, the system's knowledge is correct, but incomplete. Exhaustivity and disjunction conditions are not satisfied, because Dogs x Mammals $\neq$ Animals.

In a simple classification system, an inheritance relationship like $(X \rightarrow Y)$ must be interpreted as $\kappa(X)+(\kappa(X))^{\prime}=\kappa(Y)$. That equation may be read as "the known part of $X$ plus its complementary in the known part of $Y$ is equivalent to the known part of $Y$." Following the previous example: $\kappa($ Dogs $)+(\kappa(\text { Dogs }))^{\prime}=\kappa($ Animals $)$ and $k$ (Mammals $)+(\kappa(\text { Mammals }))^{\prime}=\kappa($ Animals $)$. The conclusion is that dogs are animals, and mammals are animals (the correct but incomplete information). It isn't a conclusion that dogs are not-mammals, though the known subclass of dogs has been included in the class of animals that are not known as mammals. It is not true that "for all $X$ and $Y, k(X)+(K(X))^{\prime}$ implies $X \subseteq Y^{\prime}$ or $Y \subseteq X^{\prime}$," but it is twe that "for all $X$ and $Y, k(X)+(k(X))^{\prime}$ $=k(Y)+(K(Y))^{\prime}$ implies $k(X) \subseteq(K(Y))^{\prime}$ and $k(Y) \subseteq(k(X))^{\prime}$." This clearly matches incomplete knowledge intuition: it is not known whether dogs are mammals or not, but this possibility is not ruled out either.

\subsection{Classification with Exceptions}

Classification with exceptions can't be modeled by utilizing additive operations. Saying that "birds are fliers, but there are exceptions such as penguins," is equivalent to making a comparison, or multiplication between two simple classifications. However, such a comparison is not reducible to a simple classification in the same way that Grouping IV is not reducible to Groupings I or II.

Indeed, consider the expression: $B$ irds + Birds $^{\prime}=U$, and Fliers + Fliers ${ }^{\prime}=U$, where $U$ is the universe. Multiplying these classifications gives a classification composed of: i) birds that fly, ii) birds that don't fly, iii) fliers that are not birds, and iv) non-fliers that are not birds.

An inheritance relation that admits exceptions such as "birds are fliers" must be interpreted as the knowledge that one has about such a compound classification. It's necessary to be explicit about which are the known subclasses. Subclasses that are not known can be interpreted as exceptionals for that relationship. Representing birds by " $B$ " and fliers by " $F$ ", the relation with exceptions among " $B$ " and " $F$ " can be interpreted as: $\left(k(B \times F=B F), k\left(B^{\prime} x F=B ' F\right), k\left(B^{\prime} x F^{\prime}=B^{\prime} F^{\prime}\right)\right\}$. Representing these unknown subclasses graphically by regions marked with 1 , and exceptional classes by regions marked with 0 , results in: 


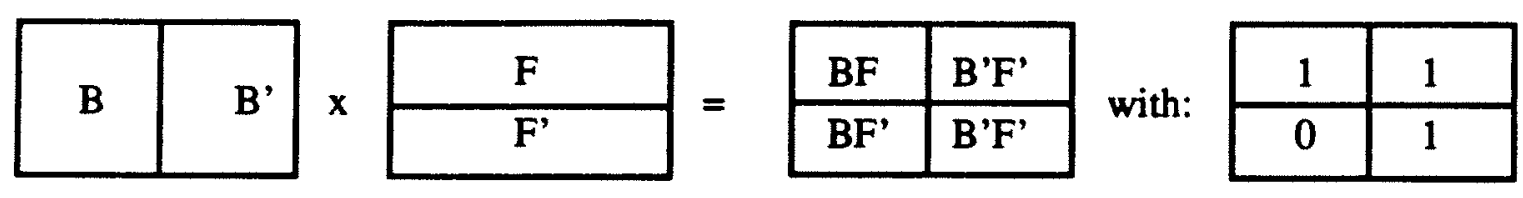

The exception to that rule (penguins are birds) can be represented by:
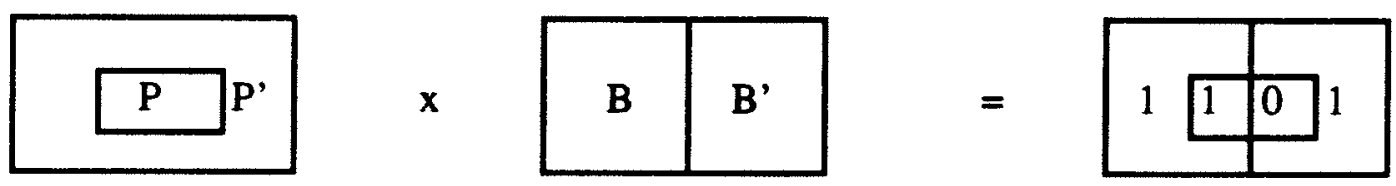

The transitive composition (penguins are birds) and (birds are fliers) can be represented by:

\begin{tabular}{|l|l|l|l|}
\hline \multirow{2}{*}{ P'BF $^{\prime}$} & \multicolumn{2}{c|}{ P'B'F } \\
\cline { 2 - 4 } & PBF & PB'F & \\
\hline P'BF' & PBF' & PB'F' & P'B'F' \\
\hline
\end{tabular}

For calculating the value of each subclass represented in the previous diagram, one must do arithmetic multiplication of values of each coincident region in the component diagrams:

$(*)$

\begin{tabular}{|l|l|}
\hline 1 & 1 \\
\hline 0 & 1 \\
\hline
\end{tabular}
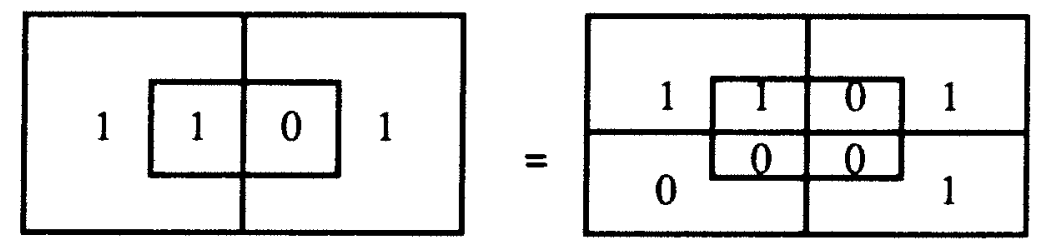

Since the only known subclass of penguins is PBF (see the inner square in the previous diagram), it follows that penguins are birds and penguins are fliers.

The contradictory information is given by "penguins are not fliers," represented by: 
$(* *)$

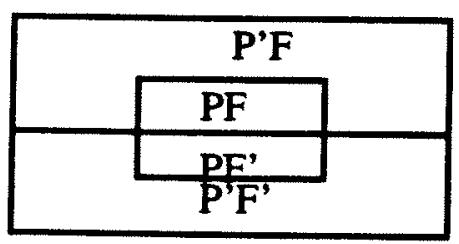

with:

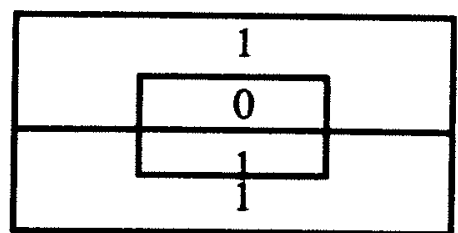

The expression of contradiction is in the composition of information "penguins are birds," "birds are fliers" and "penguins aren't fliers," represented by:
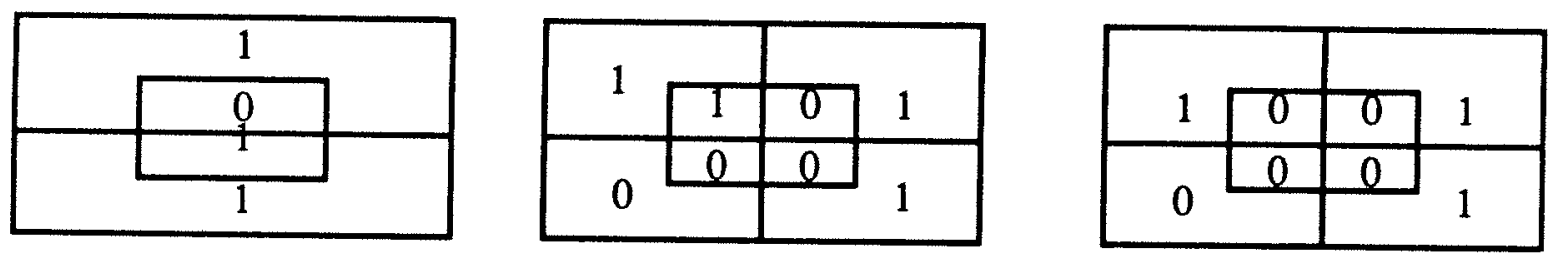

It can thus seen that every subclass of penguins is exceptional. Thus, the class of penguins itself is exceptional for that set of information.

Non-monotonic inheritance can be accomplished through a preference criterion applied to contradictory information. It is possible, for example, to choose a diagram with the smallest number of divisions (or regions). In that case, one will prefer information given by diagram $(* *)$, rather than that of $(*)$, and will conclude that penguins don't fly.

If that is chosen to be the general rule, the algorithm for inheritance searching will be like that proposed by Fahlmann [FAL79]. Thus, the shortest paths in an inheritance graph will determine which attributes will be inherited by a class.

\section{CONCLUSION}

It was shown that Jean Piaget's operative logic can be a good tool for systematizing concepts and understanding a classification system's evolution. That tool may also be useful for analyzing other cognitive structures of intelligent computational systems.

The known approaches in Artificial Intelligence talk about natural knowledge utilizing mathematical logic. However, it seems to be an interesting approach to talk about natural knowledge using a natural logic, such as operative logic. Such an approach, however, wouldn't be useful unless that natural logic were well defined. Thus, it is necessary to use a mathematical metalanguage, like first order predicate calculus, to refer to the language of natural logic. 
The language of operative logic is not yet completely formalized, because it is content dependent. That content is natural (not formal) thinking itself. Nevertheless, the author intends to publish soon a paper where he proposes a rigorous formalization of the eight groupings of intrapropositional operative logic (operative logic of classes and relations). From that formalization may arise an environment for describing relational and classificatory structures through a rich language, identified with the structures of natural thinking. That environment can also be useful for giving a semantics to knowledge representation systems, by mapping each structure of these systems in a cognitive structure of one of the eight groupings of intrapropositional operative logic.

\section{BIBLIOGRAPHY}

[FAL 79] FAHLMAN, S. NETL: A System for Representing and Using Real-World Knowledge. MIT Press, Cambridge, MA, 1979. (Op. Cit. in: Horty, J. F. and Thomason, R. H. A Skeptical Theory of Inheritance in Nonmonotonic Semantic Networks. Artificial Intelligence 42(1990): 311-48.

[PIA 71] PIAGET, Jean. Essai de Logique Operatoire. Dunod, 1971. Ensaio de Logica Operatoria. Porto Alegre, Globo, 1976. Segunda edicao do Tratado de Logica - Ensaio de Logistica Operatoria, 1949, estabelecida por Jean-Blaise Grize. Trad. por Maria A. V. de Almeida. 